\title{
Barreiras associadas à prática de atividade física no tempo livre de idosos com insuficiência cardíaca
}

\author{
Barriers associated with physical activity practice during leisure time among elderly \\ with heart failure
}

\section{AUTORES \\ Bruno Giglio de Oliveira ${ }^{1}$ (D) \\ Marilin $\mathrm{Hohl}^{1}$ (D) \\ Adriano Akira Ferreira Hino H,2,3 $^{12}$ \\ 1 Pontifícia Universidade Católica do Paraná Residência Multiprofissional em Saúde do Idoso do Hospital Santa Casa de Misericórdia de Curitiba. Curitiba, Paraná, Brasil. \\ 2 Pontifícia Universidade Católica do Paraná Programa de Pós-Graduação em Tecnologia em Saúde. Curitiba, Paraná, Brasil. \\ 3 Universidade Tecnológica Federal do Paraná. Programa de Pós-Graduação em Educação Física. Curitiba, Paraná, Brasil}

\section{CONTATO}

Bruno Giglio de Oliveira

gigliobruno@botmail.com

Rua Carlos Dietzsch, 541, Apto. 306, Portão, Curitiba, Paraná, Brasil. CEP: 80.330-000.

DOI

$10.12820 /$ rbafs. $23 \mathrm{e} 0051$

\begin{abstract}
RESUMO
O objetivo deste estudo foi analisar a associação entre as barreiras para a prática de atividade física com o nível de atividade física no tempo livre de idosos com insuficiência cardíaca. Trata-se de um estudo transversal, no qual foram analisados dados de 156 idosos (40,4\% mulheres) de 60 a 92 anos de idade de um ambulatório acadêmico de insuficiência cardíaca. Foram analisados os dados referentes a informações sociodemográficas, comorbidades, comportamento de risco, barreiras para atividade física e prática de atividade física no tempo livre. Para verificar a associação entre as variáveis foi utilizado o teste Qui-quadrado para tendência. Dentre as barreiras relatadas pelos idosos, as principais foram associadas com a condição clínica, como: falta de ar (27,4\%), fraqueza nas pernas $(21,7 \%)$ e dor nas pernas $(19,1 \%)$. O nível de atividade física no tempo livre dos idosos apresentou diferença significativa conforme o número de barreiras percebidas ( $p=0,018)$, onde idosos que relataram mais barreiras eram mais inativos fisicamente. Considerando esses achados, estratégias devem ser desenvolvidas para que haja uma educação em saúde a fim de salientar os benefícios da atividade física a longo prazo no controle das principais barreiras, para que os idosos tenham maior chance de iniciar e manter uma prática regular de atividade física.
\end{abstract}

Palavras-chave: Atividade motora; Exercício; Idoso; Doenças cardiovasculares; Insuficiência cardíaca.

\begin{abstract}
The objective of this study was to analyse the association between barriers to physical activity practice and the level of leisure physical activity among older adults with heart failure. This is a cross-sectional study, in which data from 156 elderly (40.4\% women) aged 60-92 years-old from an academic outpatient clinic of heart failure were analyzed. Data regarding sociodemographic information, comorbidities, risk behavior, barriers to physical activity and leisure time physical activity were evaluated. To verify the association between the variables, the Chi-square test for trend was used. Among the barriers reported by the elderly, the main ones were related to the clinical condition, such as: shortness of breath (27.4\%), weakness in the legs (21.7\%) and pain in the legs (19.1\%). The level of leisure time physical activity had a significant difference according to the number of perceived barriers $(p=0.018)$, demonstrating that older people who reported more barriers were more physically inactive. Because of this, strategies should be developed for health education in order to emphasize the long-term benefits of physical activity in controlling the main barriers, so that the elderly have a greater chance of starting and maintaining a regular practice of physical activity.

Keywords: Motor activity; Exercise; Aged; Cardiovascular disease; Heart failure.
\end{abstract}

Este obra está licenciado com uma Licença Creative Commons Atribuição-NãoComercialCompartilhaIgual 4.0 Internacional.

\section{Introdução}

A taxa de crescimento da população idosa no Brasil é considerada acima da média mundial. Estimativas sugerem que em 2070 a proporção da população com 60 anos ou mais seja superior aos de países desenvolvidos ${ }^{1}$. Diante deste fato, observa-se um aumento da prevalência de morbidades características da população idosa. Dentre estas doenças, as cardiovasculares apresentam grande impacto, visto que atualmente são a maior causa de morte no Brasil, e sua prevalência aumenta conforme o envelhecimento da população ${ }^{2}$. A insuficiência cardíaca (IC) é considerada a via final comum dentre a maioria das doenças cardiovasculares, sendo um dos mais importantes desafios clínicos atuais na área da saúde ${ }^{3}$. No Sistema Único de Saúde (SUS), no ano de 2017, a IC foi responsável por 205.824 internações, gerando 22.381 óbitos e um custo hospitalar de $\mathrm{R} \$$ 306.253.602,27 .

Dentre as opções de tratamento clínico da IC, a prática regular de atividade física atua diretamente na melhoria da capacidade funcional, promovendo benefícios tanto específicos para o coração quanto sistêmi- 
$\cos ^{5-12}$. Entretanto, a aderência a este tipo de tratamento é baixa ${ }^{13}$, e o fato de ser idoso é um dos principais determinantes para tall ${ }^{14}$. Entre os fatores associados a inatividade física, estudos têm consistentemente mostrado que as barreiras estão dentre os principais determinantes deste comportamento em idosos. De maneira geral, as principais barreiras para atividade física identificadas nos idosos com IC têm sido: severidade dos sintomas, sentir-se fisicamente cansado, efeitos colaterais dos medicamentos, depressão, visão negativa da vida devido a doença, entre outros ${ }^{14,15}$.

No entanto, as principais barreiras percebidas identificadas em idosos com IC se mostram distintas quando comparadas com as principais barreiras em idosos que não possuem a doença ${ }^{16,17}$. Apesar da literatura já estar bem fundamentada quanto os benefícios da atividade física para idosos com IC, estudos no Brasil não têm descrito os motivos pelos quais esta população não adere regularmente à esta prática. Ainda, o número de pesquisas que têm testado a associação entre as barreiras percebidas com o nível de atividade física no tempo livre é bastante reduzido. Considerando esse contexto, o presente estudo teve como objetivo analisar a associação entre as barreiras para a prática de atividade física com o nível de atividade física no tempo livre de idosos com IC.

\section{Métodos}

Trata-se de um estudo de delineamento transversal, baseado em dados secundários, obtidos através dos prontuários de idosos com IC de um ambulatório acadêmico de uma instituição comunitária de ensino superior da cidade de Curitiba, Paraná, Sul do Brasil.

Neste ambulatório os pacientes com IC são atendidos por uma equipe multiprofissional, constituída por professores e acadêmicos do curso de medicina, residentes da cardiologia e residentes de um programa de residência multiprofissional em saúde do idoso (Enfermagem, Educação Física, Farmácia, Fisioterapia, Nutrição, Psicologia, Odontologia e Serviço Social). São atendidos anualmente cerca de 800 pacientes adultos e idosos (40,0\% idosos) com IC, predominantemente do SUS. Esses pacientes retornam ao ambulatório durante o ano, de duas a cinco vezes para acompanhamento, de acordo com as necessidades de cada paciente.

$\mathrm{Na}$ primeira consulta, optou-se pela aplicação de um formulário de anamnese multiprofissional, de modo individualizado, em que os residentes leem as perguntas e as possíveis respostas, permitindo ao idoso indicar a opção que melhor represente as característi- cas da sua saúde e seu estilo de vida. Os pesquisadores assinaram o Termo de Compromisso de Utilização dos Dados (TCUD) e o estudo foi aprovado pelo comitê de ética em pesquisa da PUC-Paraná em 17/08/2016, parecer $n^{\circ} 1.682 .940$.

A população do estudo foi composta por todos os idosos com IC que foram atendidos pelos residentes do programa de residência multiprofissional em saúde do idoso, no período de março de 2014 a junho de 2016. O estudo teve como critérios de elegibilidade idosos com IC, de ambos os sexos, com idade igual ou superior a 60 anos, sem limitações físicas que impossibilitasse a prática de atividade física. Os prontuários que continham formulários de anamnese com informações incompletas foram excluídos. A amostra do estudo foi composta por 156 idosos com IC, entre 60 a 92 anos de idade.

O formulário foi composto por 146 perguntas fechadas e uma aberta, dividido em quatro blocos: identificação, percepções e expectativas, necessidades psicossociais e necessidades psicobiológicas. $\mathrm{O}$ formulário tem como finalidade registrar todas as informações importantes, permitindo uma avaliação clínica e uma orientação/intervenção educativa mais efetiva e direcionada aos idosos com IC. Inicialmente, os idosos eram levados individualmente a um consultório do ambulatório acadêmico com a presença de dois residentes, os quais se dividiam entre realizar as perguntas do formulário com as possíveis respostas e preencher as respostas. A duração da aplicação do formulário era em média de 30 minutos, e após eram realizadas orientações quanto as necessidades encontradas mediante as respostas.

A avaliação das barreiras para atividade física foi realizada por meio da pergunta "Quais os motivos que dificultam você a praticar atividade física?”, contendo 23 alternativas entre barreiras fisiológicas, psicossociais e sociodemográficas, com respostas de "sim" e "não" para cada barreira questionada. Este instrumento foi desenvolvido de maneira específica para idosos, considerando as especificidades da IC. As respostas foram dicotomizadas a fim de facilitar a compreensão dos idosos, principalmente com baixa escolaridade. Tal adaptação já foi utilizada em um estudo no Brasil com idosos, também no domínio de atividade física no tempo livre ${ }^{18}$.

Dentre as características sociodemográficas, foi registrado o sexo ("masculino" e "feminino") e a idade em anos classificada em faixas etárias de "60-69 anos", "7079 anos" e "80 anos". Peso e estatura foram autorreportados e posteriormente utilizados para determinar o índice de massa corporal (IMC), o qual categorizado 
segundo classificação específica para idosos ${ }^{19}$ (“< $<22 \mathrm{~kg}$ / $\mathrm{m}^{2}$ ", "22- $27 \mathrm{~kg} / \mathrm{m}^{2}$ ” e > " $27 \mathrm{~kg} / \mathrm{m}^{2 ”)}$. Renda domiciliar foi autorreportada e classificada em "até 1 salário mínimo", "2-3 salários mínimos" e " $\geq 4$ salários mínimos". Grau de instrução foi autorreportado, sendo possível o participante relatar que iniciou e/ou completou os diferentes níveis que foram classificados em "analfabeto", "ensino fundamental", "ensino médio" e "ensino superior".

Entre as características de saúde, seis comorbidades (hipertensão, dislipidemia, diabetes, insuficiência renal crônica, doença pulmonar obstrutiva crônica e artrose) foram avaliadas com a questão "Você possui algum destes problemas de saúde?” que eram reportados pelo avaliador e o participante tinha opção de resposta "sim" e "não" para cada condição clínica. A dispneia ao esforço foi avaliada por meio da questão "Você sente falta de ar quando realiza algum tipo de esforço?" e foi classificada em "não sente", "aos mínimos esforços", "aos moderados esforços" e "aos esforços intensos".

A atividade física em uma semana habitual foi avaliada por meio do módulo de lazer do International Physical Activity Questionnaire $e^{20}$ (IPAQ), traduzido, adaptado e validado para utilização em idosos no Brasil $^{21}$. Os participantes relataram o tipo de atividade física, número de dias por semana e tempo por dia em atividades físicas no tempo livre. $\mathrm{O}$ nível de atividade física no tempo livre foi classificado em "fisicamente inativo" e "fisicamente ativo". Para caracterizar os idosos como fisicamente ativos, eles deveriam somar pelo menos 150 minutos de atividade física no tempo livre em uma semana habitual, conforme a recomendação da Organização Mundial de Saúde (OMS) 22 .

Os dados do presente estudo foram descritos por meio da distribuição de frequências (absoluta e relativa). Para verificar a associação do número de barreiras percebidas com o nível de atividade física no tempo livre, foi utilizado o teste Qui-quadrado para tendência. $\mathrm{O}$ nível de significância adotado foi de $\mathrm{p} \leq 0,05$. Todas as análises foram realizadas com auxílio do pacote estatístico SPSS versão 20.0.

\section{Resultados}

A população do estudo foi composta por 189 idosos com IC. Houve perda de 17,5\% dos dados dos formulários por apresentarem informações incompletas, tendo como amostra final um total de 156 formulários completos.

Em relação as variáveis sociodemográficas, houve maior proporção de idosos entre 60-69 anos (58,3\%), do sexo masculino (59,6\%), com IMC classificado como acima do peso $(53,2 \%)$, renda domiciliar entre 2-3 salários mínimos $(42,3 \%)$ e ensino fundamental iniciado e/ou completo como grau de instrução mais relatado (66,7\%) - Tabela 1.

Quanto às variáveis relacionadas à saúde, houve maior e menor proporção de hipertensão arterial $(84,6 \%)$ e doença pulmonar obstrutiva crônica (DPOC) $(5,8 \%)$, respectivamente. Maior proporção dos idosos relataram não sentir dispneia aos esforços $(47,4 \%)$ e foram considerados fisicamente inativos no tempo livre $(79,5 \%)$ - Tabela 1.

Tabela 1 - Características descritivas dos participantes. Curitiba, Paraná, Brasil, 2014-2016 ( $\mathrm{n}=156)$.

\begin{tabular}{|c|c|c|}
\hline Variáveis & $\mathrm{n}$ & $\%$ \\
\hline \multicolumn{3}{|l|}{ Sexo } \\
\hline Feminino & 63 & 40,4 \\
\hline Masculino & 93 & 59,6 \\
\hline \multicolumn{3}{|l|}{ Faixa etária } \\
\hline 60 a 69 anos & 91 & 58,3 \\
\hline 70 a 79 anos & 42 & 26,9 \\
\hline$\geq 80$ anos & 23 & 14,7 \\
\hline \multicolumn{3}{|l|}{ IMC } \\
\hline Baixo peso & 15 & 10,8 \\
\hline Peso adequado & 50 & 36,0 \\
\hline Acima do peso & 74 & 53,2 \\
\hline Renda domiciliar & 57 & 36,5 \\
\hline \multicolumn{3}{|l|}{ Até um salário mínimo } \\
\hline Dois a três salários mínimos & 66 & 42,3 \\
\hline Quatro salários mínimos ou mais & 33 & 21,2 \\
\hline Grau de instrução & 27 & 17,3 \\
\hline \multicolumn{3}{|l|}{ Analfabeto } \\
\hline Ensino fundamental & 104 & 66,7 \\
\hline Ensino médio & 18 & 11,5 \\
\hline Ensino superior & 7 & 4,5 \\
\hline \multicolumn{3}{|l|}{ Comorbidades reportadas } \\
\hline DPOC & 9 & 5,8 \\
\hline Insuficiência renal crônica & 12 & 7,7 \\
\hline Artrose & 15 & 9,6 \\
\hline Diabetes & 38 & 24,4 \\
\hline Dislipidemia & 42 & 26,9 \\
\hline Hipertensão arterial & 132 & 84,6 \\
\hline \multicolumn{3}{|l|}{ Dispneia } \\
\hline Não sente & 74 & 47,4 \\
\hline Aos mínimos esforços & 24 & 15,4 \\
\hline Aos moderados esforços & 51 & 28,8 \\
\hline Aos esforços intensos & 7 & 4,5 \\
\hline \multicolumn{3}{|l|}{ Nível de atividade física no tempo livre } \\
\hline Fisicamente ativos & 32 & 20,5 \\
\hline Fisicamente inativos & 124 & 79,5 \\
\hline
\end{tabular}

IMC = Índice de massa corporal; DPOC = Doença pulmonar obstrutiva crônica. 
Em relação às barreiras para a prática de atividade física relatadas pelos idosos, houve maior proporção de falta de ar $(27,4 \%)$ e fraqueza nas pernas $(21,7 \%)$, e menor proporção de falta de segurança no bairro $(0,6 \%)$ e falta de dinheiro (0,6\%) - Figura 1 . Dentre os idosos que relataram realizar algum tipo de atividade física no tempo livre, houve maior proporção de caminhada $(41,8 \%)$ e não houve nenhum relato de prática de musculação (Figura 2).

$\mathrm{Na}$ análise de associação entre o número de barreiras

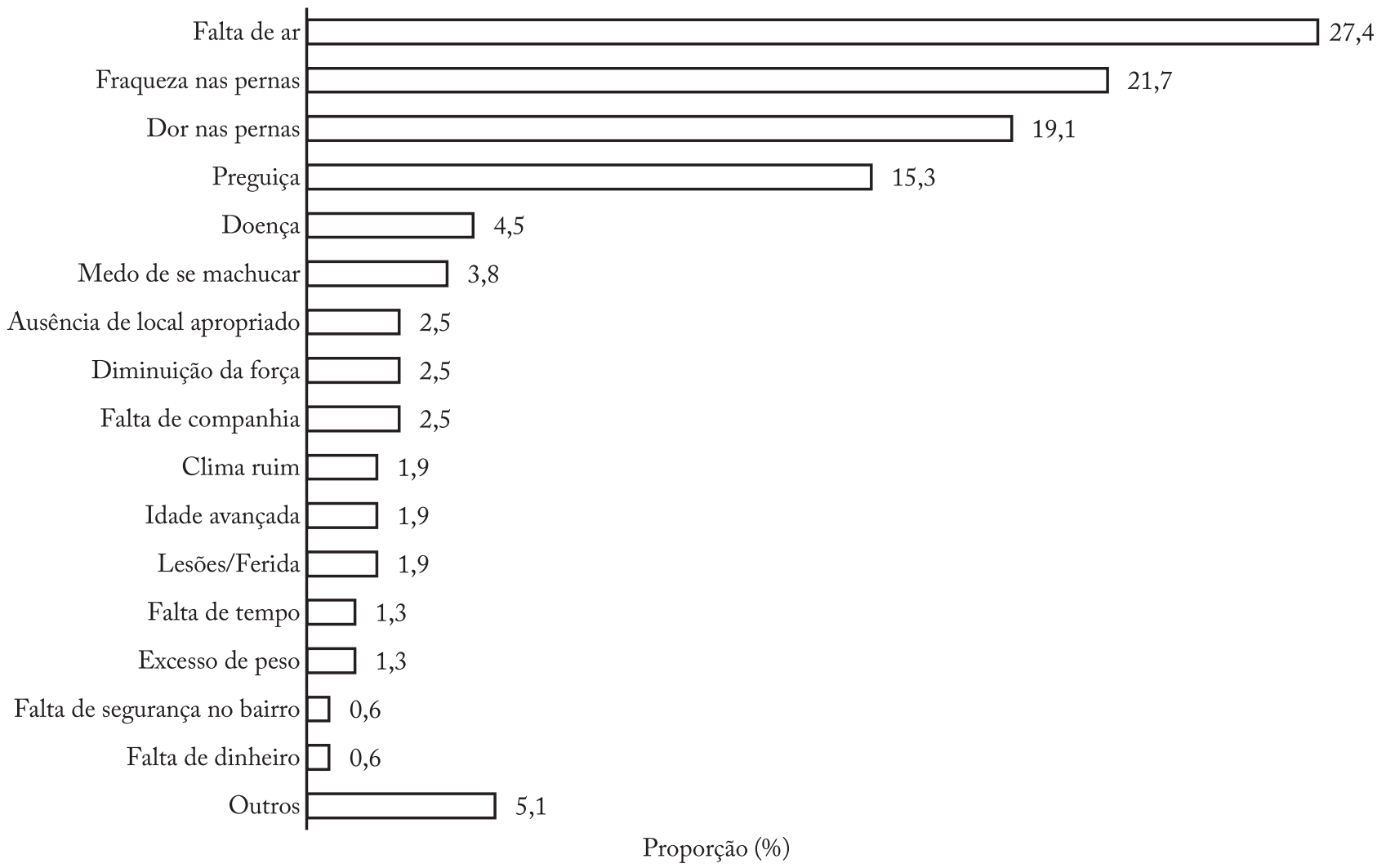

Figura 1 - Barreiras para a prática de atividade física relatadas pelos idosos com insuficiencia cardíaca. Curitiba, Paraná, Brasil, 2014-2016 ( $\mathrm{n}=156$ ).

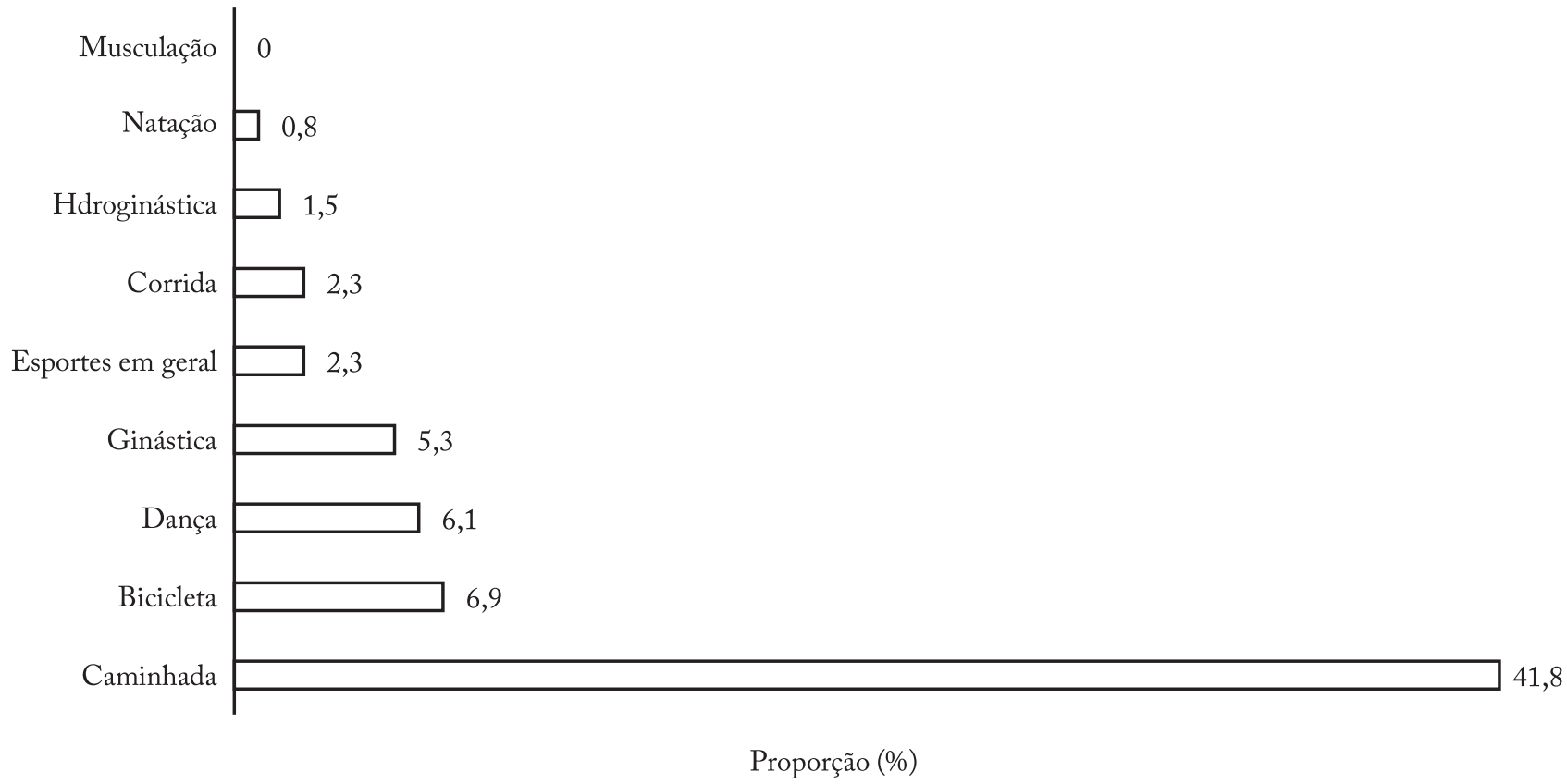

Figura 2 - Tipos de atividades físicas relatadas pelos idosos com insuficiência cardíaca. Curitiba, Paraná, Brasil. $2014-2016$ (n = 103). 
percebidas e o nível de atividade física no tempo livre dos idosos, verificou-se uma associação estatisticamente significante $(\mathrm{p}=0,018)$ e inversa entre as variáveis. Tal associação indicou que, quanto maior o número de barreiras percebidas, menor foi o nível de atividade física no tempo livre entre os idosos com IC (Figura 3).

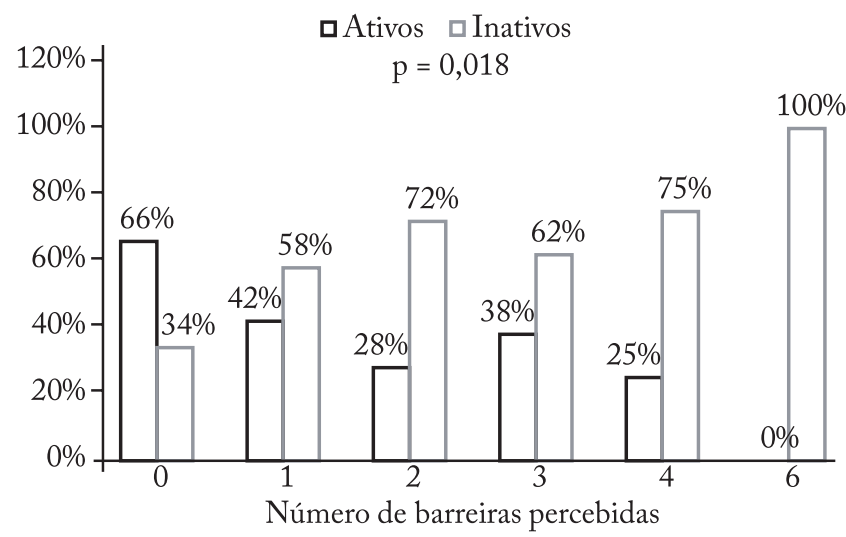

Figura 3 - Associação entre número de barreiras percebidas com o nível de atividade física no tempo livre de idosos com insuficiência cardíaca. Curitiba, Paraná, Brasil, 2014-2016 ( $\mathrm{n}=156$ ).

\section{Discussão}

A combinação entre a $\mathrm{IC}$ e seus principais sintomas contribui de maneira negativa para a prática regular de atividade física, especialmente em idosos, pelo fato deste grupo etário apresentar uma menor capacidade funcional e maior chance de apresentar dispneia aos esforços, consequentemente, aumentando a chance de se tornarem idosos fisicamente inativos ou desabilitados funcionais ${ }^{23}$. Estudos fora do Brasil apresentam resultados similares quanto as dimensões das barreiras encontradas, mostrando que a condição física do idoso é um fator prevalente e que contribui de maneira negativa para a prática regular de atividade física ${ }^{14,15}$. Um estudo realizado na Suécia ${ }^{15}$ indicou uma prevalência de $82 \%$ e $81 \%$ respectivamente, nas barreiras "sentindo-se fisicamente cansado" e "presença de sintomas".

As principais barreiras para a atividade física relatadas pelos idosos foram relacionadas aos problemas gerados pela IC, o que indica que há um desconhecimento não só dos benefícios que a atividade física pode trazer, mas também, acerca de como estes idosos podem ser ativos com a doença. De fato, estas informações não são repassadas de maneira efetiva pelos profissionais de saúde, e isso causa um receio entre os idosos em realizar qualquer tipo de atividade física, pois acreditam que a prática leva a piora dos sintomas da doença ${ }^{24}$. Estes problemas são tratados como desafios por uma diretriz reconhecida sobre o tema, entretanto, não há no docu- mento orientações de possíveis soluções ${ }^{25}$.

Apesar de observadas várias barreiras que dificultam a adesão de idosos com IC a prática regular de atividade física, algumas atividades são mais prevalentes que outras. Evidenciou-se no presente estudo, que aproximadamente $42 \%$ de idosos realizam caminhadas, o que sugere que há uma maior prevalência em atividades que não necessariamente precisam de um espaço particular e de custo para serem realizadas. De fato, um estudo indicou que entre indivíduos portadores de IC, a prática de caminhada é a mais realizada, entretanto, ainda abaixo da quantidade necessária para atingir a recomendação da OMS. Mais que isso, indivíduos com esta condição gastam em média 14 vezes mais tempo sentados, caracterizando um comportamento sedentário, quando comparado ao tempo caminhando no tempo livre ${ }^{26}$.

Apesar dos avanços no tratamento da IC, em idosos ainda permanece crescente a incidência de novos casos desta condição, mesmo em países desenvolvidos ${ }^{27}$, e, somado este fato ao aumento da expectativa de vida da população, espera-se que idosos vivam mais tempo com os efeitos deletérios da doença ${ }^{27}$. Com base nisso, uma maior capacidade funcional mostra-se fundamental para uma melhor qualidade de vida dos idosos ao longo do tempo.

Diante destes elementos, estratégias bem definidas são necessárias para contribuir com a melhora da adesão de idosos com IC em programas regulares de atividade física. Evidências sugerem que a prescrição correta do exercício seguindo as diretrizes de instituições reconhecidas, monitoramento via telefone, educação em saúde, aconselhamento e estratégias motivacionais pode contribuir para esta melhora em curto prazo, porém, ainda permanece inconsistente a forma de manter a aderência ao longo do tempo ${ }^{27-30}$.

Como limitações do presente estudo, o formulário utilizado não é um instrumento validado, o que implica em possíveis vieses pelo fato das perguntas serem fechadas e de múltipla escolha, não possibilitando investigar de maneira aprofundada o constructo. Também, não contém no formulário a classificação funcional da IC, o que deixa mais subjetivo os resultados das barreiras percebidas e nível de atividade física, por não haver a associação entre a classe funcional e estas variáveis. Tanto as barreiras percebidas quanto as atividades exercidas pelos idosos foram classificadas de forma objetiva, onde já havia previamente as opções em múltipla escolha e cabia ao profissional investigá-las, tornando o estudo com um caráter quantitativo, sem 
aprofundar nos detalhes das barreiras percebidas e das atividades realizadas. Por fim, o nível de atividade física foi investigado de maneira subjetiva e avaliado apenas no domínio do lazer.

Como pontos fortes, o formulário envolvendo múltiplas variáveis colabora na descrição dos indivíduos, ajudando os leitores a entenderem o perfil estudado. Além disso, o local do estudo é um local onde intervenções podem ser realizadas com base neste resultado, e são sugeridas ao longo do presente estudo estratégias desenvolvidas por outros locais para intervenção, que podem ser reproduzidas no Brasil.

Conclui-se que os idosos com IC apresentam inúmeras barreiras que podem implicar em torná-los inativos fisicamente, com uma baixa capacidade funcional e consequentemente uma menor qualidade de vida. De acordo com os resultados obtidos no presente estudo, pode-se inferir que a maioria dos idosos foi considerada fisicamente inativa, e que quanto mais barreiras eles apresentam, menor o nível de atividade física no tempo livre. Apesar das barreiras mais prevalentes estarem relacionadas à condição clínica devido a IC, outros fatores multidimensionais contribuem para o acúmulo de barreiras. Mesmo contendo atividades de maior facilidade para os idosos, como a caminhada, a qual foi identificada como sendo a mais realizada, a maioria dos idosos não adere a uma prática regular de atividade física.

\section{Conflito de interesses}

Os autores declaram não haver conflito de interesses.

\section{Contribuição dos autores}

Oliveira BG, concepção inicial do estudo, análise estatística, interpretação dos dados e redação do trabalho. Hohl M, participou do desenho do estudo, revisão e aprovação final do estudo. Hino AAF, participou do desenho do estudo, análise estatística, interpretação dos dados e aprovação final do estudo.

\section{Referência}

1. IBGE - Instituto Brasileiro de Geografia e Estatística. Síntese de Indicadores Sociais - Uma análise das condições de vida da população brasileira. Brasil; 2016.

2. IBGE - Instituto Brasileiro de Geografia e Estatística. Pesquisa Nacional de Saúde: percepção do estado de saúde, estilos de vida e doenças crônicas - Brasil, Grandes Regiões e Unidades da Federação [Internet]. Rio de Janeiro: Instituto Brasileiro de Geografia e Estatística; 2014. [citado $2018 \mathrm{fev}$ 09]. Disponível em: url: ftp://ftp.ibge.gov.br/PNS/2013/ pns2013.pdf.

3. Bocchi EA,Marcondes-Braga FG, Ayub-Ferreira SM, Rohde LE, Oliveira WA, Almeida DR, et al. Sociedade Brasileira de Cardiologia. III Diretriz Brasileira de Insuficiência Cardíaca Crônica. Arq Bras Cardiol. 2009;93(Suppl1):1-71.
4. Brasil, Ministério da Saúde. Banco de dados do Sistema Único de Saúde-DATASUS. [citado 2018 fev 09]. Disponível em: url: http://www.datasus.gov.br.

5. Gielen S, Laughlin MH, O'Conner C, Duncker DJ. Exercise training in patients with heart disease: Review of beneficial effects and clinical recommendations. Prog Cardiovasc Dis. 2015;57(4):347-55.

6. Flynn KE, Pinã IL, Whellan DJ, Lin L, Blumenthal JA, Ellis SJ, et al. Effects of exercise on health status in patients with chronic heart failure: Findings from the HF-ACTION randomized controlled trial. JAMA. 2009;8;301(14):1451-9.

7. Arena R, Cahalin LP, Borghi-Silva A, Phillips SA. Improving functional capacity in heart failure: the need for a multifaceted approach. Curr Opin Cardiol. 2014;29(5):467-74.

8. Laoutaris ID, Adamopoulos S, Manginas A, Panagiotakos DB, Kallistratos MS, Doulaptsis C, et al. Benefits of combined aerobic/resistance/inspiratory training in patients with chronic heart failure. A complete exercise model? A prospective randomised study. Int J Cardiol. 2013;167(5):1967-72.

9. Izawa KP, Watanabe S, Oka K, Hiraki K, Morio Y, Kasahara $\mathrm{Y}$, et al. Association between mental health and physical activity in patients with chronic heart failure. Disabi Rehabil. 2014;36(3):250-4.

10. Ozasa N, Morimoto T, Bao B, Shioi T, Kimura T. Effects of machine-assisted cycling on exercise capacity and endothelial function in elderly patients with heart failure. Circ J. 2012;76(8):1889-94.

11. Haykowsky MJ, Kitzman DW. Exercise physiology in heart failure and preserved ejection fraction. Heart Fail Clin. 2014;10(3):445-52.

12. Reiter BD, Arora RR. The role of exercise in heart failure: a systematic review. Am J Ther. 2014;21(5):403-11.

13. van der Wal MH, van Veldhuisen DJ, Veeger NJ, Rutten FH, Jaarsma T. Compliance with non-pharmacological recommendations and outcome in heart failure patients. Eur Heart J. 2010;31(12):1486-93.

14. Klompstra L, Jaarsma T, Strömberg A. Physical activity in patients with heart failure: barriers and motivations with special focus on sex differences. Patient Prefer Adherence. 2015;9(9):1603-10.

15. Tierney S, Elwers H, Sange C, Mamas M, Rutter MK, Gibson M, et al. What influences physical activity in people with heart failure? a qualitative study. Int J Nurs Stud. 2011;48(10):1234-43.

16. Cassou ANC, Fermino RC, Santos MS, Rodriguez-Añez $\mathrm{CR}$, Reis RS. Barreiras para à atividade física em idosos: uma análise por grupos focais. Rev Educ Fis. 2008;19(3): 353-60.

17. Buman MP, Yasova LP, Giacobbi Jr PR. Descriptive and narrative reports of barriers and motivators to physical activity in sedentary olders adults. Psych Sport Exer. 2010;11(3):223-230.

18. Salvador EP, Florindo AA, Reis RS, Costa EF. Percepção do ambiente e prática de atividade física no lazer entre idosos. Rev Saúde Pública. 2009;43(6):972-80.

19. Souza R, Fraga JS, Gottschall CBA, Busnello FM, Rabito EI. Avaliação antropométrica em idosos: estimativas de peso e altura e concordância entre classificações de IMC. Rev Bras Geriatr Gerontol. 2013;16(1):81-90.

20. Craig CL, Marshall AL, Sjostrom M, Bauman AE, Booth $\mathrm{ML}$, Ainsworth BE, et al. International physical activity questionnaire: 12 -country reliability and validity. Med Sci Sports Exerc. 2003;35(8):1381-95.

21. Mazo GZ, Benedetti TRB. Adaptação do questionário internacional de atividade física para idosos. Rev Bras Cineantropom Desempenho Hum. 2010;12(6):480. 
22. World Health Organization (WHO). Global strategy on diet, physical activity and health. Physical activity and older adults, 2011. [internet] [citado 2017 dez 05]. Disponível em: url: http://www.who.int/dietphysicalactivity/factsheet_ olderadults/en/

23. Yonamine CY, Matsuo $\mathrm{T}$, de Souza RKT, Loch MR, Cabrera MAS. Functional disability for mobility in adults: a population-based study. Fisioter Mov. 2016;29(3):449-59.

24. Albert NM, Forney J, Slifcak E, Sorrell J. Understand physical activity and exercise behaviors in patients with heart failure. Heart Lung. 2015;44(1):2-8.

25. Herdy AH, López-Jimenez F, Terzic CP, Milani M, Stein R, Carvalho T; Sociedade Brasileira de Cardiologia. Diretriz Sul-Americana de Prevenção e Reabilitação Cardiovascular. Arq Bras Cardiol 2014; 103(2Supl.1): 1-31.

26. Doukky R, Mangla A, Ibrahim Z, Poulin MF, Avery $\mathrm{E}$, Collado FM, et al. Impact of physical inactivity on mortality in patients with heart failure. Am J Cardiol. 2015;1;117(7):1135-43.
27. Conrad N, Judge A, Tran J, Mohseni H, Hedgecott D, Crespillo AP, et al. Temporal trends and patterns in heart failure incidence: a population-based study of 4 million individuals. Lancet. 2018;391(10120):572-80.

28. American College of Sports Medicine, Chodzko-Zajko WJ, Proctor DN, Fiatarone Singh MA, Minson CT, Nigg $\mathrm{CR}$, et al. American College of Sports Medicine position stand. Exercise and physical activity for older adults. Med Sci Sports Exerc. 2009;41(7):1510-30.

29. Chan M, Tsuyuki R. Heart failure in the elderly. Curr Opin Cardiol. 2013;28(2):234-41.

30. Powell LH, Calvin Jr JE, Richardson D, Janssen I, de Leon CFM, Flynn KJ, et al. Self-management counseling in patients with heart failure: primary results from the heart failure adherence and retention trial (HART). JAMA. 2010;304(12):1331-8.

Recebido: $11 / 10 / 2018$

Aprovado: 04/03/2019

\section{Como citar este artigo:}

Oliveira BG, Hohl M, Hino AA. Barreiras associadas à prática de atividade física no tempo livre de idosos com insuficiência cardiaca. Rev Bras Ativ Fís Saúde. 2018;23:e0051.DOI: 10.12820/rbafs.23e0051 